Editorial

\title{
Occupational cancer in the oncogene era
}

The first recognition of an occupationally induced cancer is generally attributed to Sir Percivall Pott who in 1775 described scrotal cancer among London chimney sweeps. Since then, numerous observations of animal and microbial exposures, human case reports, disease clusters, and studies in occupational and environmental epidemiology have identified many proved, probable, and suspected carcinogens. Quantitatively, chemicals from smoking and occupation have been said to contribute to as much as $40 \%$ of our cancers. ${ }^{1}$

Although our understanding of the causal pathways linking dose to effect in occupational cancer has lagged behind our recognition of exposure risks, recent developments in molecular biology are quickly closing this gap. Whereas almost two centuries passed between Pott's observations and the era of molecular biology introduced by Crick and Watson in 1953, there has been less than two decades between the latter's DNA double helix proposal and the concept of "oncogenes" as put forth by Huebner and Todaro in $1969 .^{2}$ The subsequent progress of molecular oncology over the past two decades can only be described as explosive.

As a result of these rapid developments, occupational medicine now must consider both epidemiological descriptive and biochemical mechanistic models of occupational carcinogenesis. There is a considerable degree of sophistication across this spectrum of understanding as some time spent delving into the fields of occupational epidemiology and chemical genotoxicity will show. As we enter our second century of DNA research (given that Friedrich Miescher discovered nuclein in 1869) and our third century of occupational cancer epidemiology, we are finding these disciplines converging in what is becoming known as "molecular cancer epidemiology." 3 This new biology of oncogenes and antioncogenes (tumour suppressor genes) promises to be both a challenge to our future practice of occupational medicine and an opportunity for collaborative studies by molecular biologists, clinicians, and epidemiologists. ${ }^{4}$ Practitioners of occupational medicine will need to be conversant across this range of expertise in order to take part in such multidisciplinary approaches to occupational cancer in the oncogene era.

The historical roots of the oncogene era may be found at the turn of this century when Peyton Rous made his modest but far reaching discovery of a transmissible sarcoma in chickens. ${ }^{5}$ The Rous sarcoma virus (RSV), as the causative organism became known, was one of the first infectious oncogenic agents to be described and it has set the stage for much of our current knowledge of retroviral oncogenes. Fortuitously, RSV was able to replicate without a helper virus and was easily subjected to deletional mutations that enabled it to lead researchers into the mysteries of retroviral gene structure and replication.

Among the RNA containing viruses, retroviruses are distinguished by their unique reverse transcriptase enzyme, an RNA dependent DNA polymerase. The humble retrovirus proved to be a boon to our understanding of carcinogenesis by introducing us to the world of oncogenes and (onco)gene regulation. As George and Eva Klein put it, "Thanks to the recombinational accidents of the retroviral lifestyle, we now have access to the dominantly acting oncogenes as an unexpected gift from viral oncology." 6

The diminutive nine kilobase genome of the RSV was found to carry a single gene responsible for its ability to initiate and maintain neoplastic transformation. This sarcoma producing gene was called "src" (pronounced "sarc" for SaRC-oma) and it has become the prototype oncogene-that is, a genetic element that contributes to the neoplastic transformation of cells. In some cases an oncogene may be inserted into the cellular genome by a retrovirus using its reverse transcriptase (whence the gene has a prefix denoting its viral source, for example, " $v$-src").

The origin of a virally borne oncogene or v-onc such as v-src perplexed researchers until it was discovered that complementary DNA or "cDNA" probes of the RSV src gene were found to hybridise with normal cellular DNA from many vertebrates and even unicellular organisms. This surprising finding suggested that src genes are ubiquitous in 
nature (highly conserved) and are components of the normal cellular genome with important functions in non-neoplastic cellular processes. Further cDNA analysis of other retroviral oncogenes showed that homologous cellular counterparts of $\mathbf{v}$-oncs are present in normal cells. The pioneering work of Bishop and Varmus in showing the cellular character of oncogenes has recently been recognised by their Nobel prize in medicine.

To distinguish the normal "socialised" cellular genes from their oncogenic or "delinquent" variants, the former became known as proto-oncogenes. Under certain conditions such as exposure to carcinogens or chromosomal translocations, protooncogenes may be changed into cellular oncogenes or c-oncs and neoplastic transformation can result.

In 1978 the $\mathrm{v}$-src oncogene product, known as p60 v-src ("p" denotes a protein, "60" refers to its molecular weight in kiloDaltons, and the superscript denotes the encoding gene), was found to catalyse the addition of a phosphate molecule to other cellular proteins. ${ }^{7}$ Enzymes that phosphorylate proteins are called protein kinases, and Hunter has compared these to cellular transistors that act as amplifiers and switches in the biochemical circuitry of cells. ${ }^{8}$ Rather than phosphorylating the serine or threonine amino acids of proteins, however, as was the case for other known protein kinases, p60 ${ }^{\mathrm{v}-\mathrm{src}}$ was found to add phosphate to tyrosine, the only other amino acid with a hydroxyl group. Since the normal cell contains less than $1 \%$ of its protein phosphate bound to tyrosine, this surprising tyrosine specificity prompted a search for similar activity in other oncoproteins, and a diverse group of protein tyrosine kinases or PTKs are now considered members of the Src family-for example, the oncoproteins of abl, fgr, fyn, hck, lck, lyn, and yes (figure).

Oncogenes and their associated oncoproteins often have a cryptic nomenclature that is usually derived from the retroviruses or tumours in which they were first identified. For example, the "ras" oncogene was first found in RA-t S-arcoma viruses and the recently discovered "jun" oncogene was so named for its discovery in Avian Sarcoma Virus-17, the Japanese word for 17 being "JU-Nana."

The ras oncogene family is one of the most interesting models of oncogene activation in terms of occupational cancers, the three prototype members of the ras family being $\mathrm{H}$-ras (Harvey ras), K-ras (Kirsten ras), and $\mathrm{N}$-ras (neuroblastoma ras). Ras oncogenes appear to have an important role in carcinogen induced tumours and have been frequently found in spontaneous human and animal neoplasms. These genes all express a 189 amino acid protein of 21 kiloDalton molecular weight, p21 ${ }^{\text {ras }}$, which is attached at its C-terminus to the inner side of the cell membrane. Although lacking protein kinase activity, p21 ${ }^{\text {ras }}$ appears to act also as a

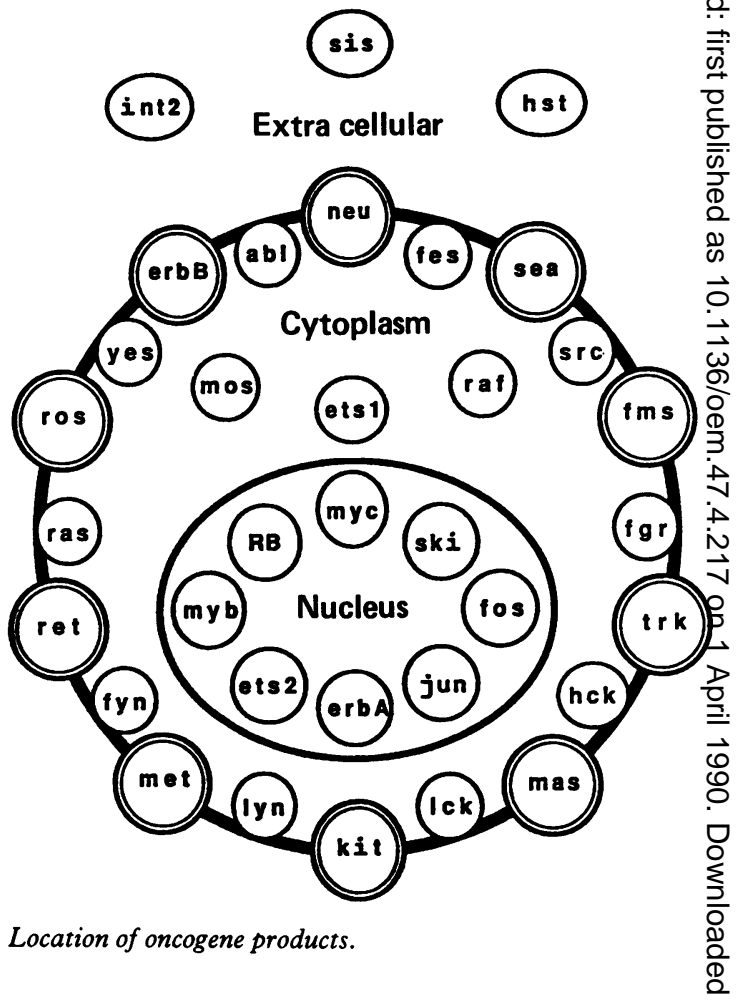

molecular switch in signal transduction by avidly $\overrightarrow{\vec{O}}$ binding GTP ("on" state) or GDP ("off" state). ${ }^{9}$ As $\exists$ p $21^{\text {ras }}$ cycles between its active and inactive states? through its intrinsic GTPase activity, it sends an: intracellular signal into the cell, possibly via theo phosphatidylinositol pathway.

Ras proteins are the first oncoproteins in whicho crystal structures have been determined. The result 3 . ing high resolution three dimensional conformation has shown atomic interactions in the single guanine? nucleotide pocket of $\mathrm{p} 21^{\text {ras }}$ where the well establishedo oncogenic point mutations at amino acids 12,13 , and 61 are found. ${ }^{10}$ These mutations do not significantly음 affect Ras GTP/GDP binding but they decrease the p2 $21^{\text {ras }}$ GTPase activity. Hydrolysis of $\mathrm{p} 21^{\text {ras }}$ boundo GTP is normally accelerated more than 100 -fold by an GTPase activating protein called GAP whichN interacts with an effector loop containing amino acids ${ }_{\sigma}^{\omega}$ 32 to 40 in p21 ${ }^{\text {ras }}$ GTP. A phosphate binding loop of amino acids 8 to 15 binds the guanine nucleotide's $\beta$ 등 phosphate and has been found to be wider in thes transforming $\mathrm{p} 21^{\text {ras }}$ which may explain the diminished GTPase activity in the oncogenic Ras. ${ }^{11}$

Experimentally, nitrosomethylurea induced mam- $-\stackrel{\vec{\Phi}}{\circ}$ mary cancer in rats produces a consistent $G$ to $A \frac{?}{\mathbb{D}}$ transition at the second nucleotide of the $\mathrm{H}$-ras codon $\frac{\varrho}{\sigma}$ 12 (where GGA normally encodes glycine) whereas dimethylbenz(a)anthracene mutates the two adenineo residues of the $\mathrm{H}$-ras codon 61 (where CAA normally 
encodes glutamine). Ras oncogenes may thus provide several mutational "hot spots" for chemical carcinogens that result in constitutively activated Ras oncoproteins and unregulated oncogenic signalling as noted above.

Ras proteins represent only a subset of the greater than 20 small GTP binding proteins that include the heterotrimeric $G$ proteins that bind GTP on their $\alpha$ subunit. Human pituitary tumours have been found to contain point mutations of the stimulatory $G$ protein $\alpha$ chain, $G_{x s}$, causing constitutive activation with on regulation of an adenylyl cyclase pathway resulting in increased synthesis of cyclic AMP.${ }^{12} \mathrm{Like}$ Ras mutations, the GTPase activity of $G_{s}$ is inhibited. The putative oncogene is to be known as "gsp" (pronounced "gasp" for $G_{s}$ protein) and promises to be the first of a new family of oncogenes that will contribute another dimension to cellular mischief. In any case, Ras and Ras related proteins have shown that GTP binding and hydrolysis is as ubiquitous a regulatory control mechanism as protein tyrosine phosphorylation.

After the discovery of the tyrosine kinase activity of the Src oncoprotein, further investigations showed a family of oncogene derived protein products having both cell surface receptors and intrinsic protein tyrosine kinase activity. These molecules are known as receptor tyrosine kinases or RTKs and include the oncogenes known as erbB-1, fms, kit, mas, met, neu, ret, ros, and trk (figure). They have a common architecture consisting of an extracellular ligand binding domain (receptor) of 500 to 850 amino acids, a transmembrane domain of 20 to 30 amino acids and an intracellular domain of 500 to 600 amino acids containing a catalytic (tyrosine kinase) site.

Such an integrated receptor catalytic structure suggests that the oncogenic potential of these molecules is due to their ability to generate a mitogenic signal. Such a signal may be produced because of $(a)$ overexpression of RTKs resulting from oncogene dysregulation or amplification (as shown for the neu oncogene in human breast cancer), (b) mutation of the ligand binding domain so as to produce conformational activation of the catalytic site in the absence of ligand (as shown for the erbB oncoprotein), or (c) mutation of the transmembrane or cytoplasmic domain so as to activate constitutively the catalytic site (as shown for the fms oncoprotein).

The receptor domains of some oncogene derived RTKs are homologous to known receptors for growth factors or other biological messengers. For example, the erbB oncogene encodes a truncated receptor for epidermal growth factor (EGF) and the mas oncogene product contains an angiotensin receptor. We have also discovered that cells may become "turned on" by so called autocrine feedback when the cell detects its own growth factors and triggers its own transformation. Oncogenes may present the cellular switches for such a process to occur.

Within the past few years much attention has focused on the role of oncogenes and their oncoproteins in cellular gene regulation. In particular, research on the control of gene transcription and cell growth has converged on the jun oncogene and its role in nuclear signalling. ${ }^{13}$

It appears that the discovery of jun, a "nuclear" oncogene, has many parallels to that of src, a "cytoplasmic" oncogene. Like src, jun was found to be the transforming sequence in an avian retrovirus and, like src, jun may produce sarcomas in chickens. Just as src proved to be the prototype oncogene, jun may become the prototype transcription regulating oncogene. Gene regulation is accomplished by cis acting sequences called promoters. A nucleotide sequence ( $5^{\prime}$ TGACTCA $3^{\prime}$ ) in certain gene promoters recognises the human transcription factor family known as AP-1. AP-1 binding to DNA is essential for such promoter activity and, rather unexpectedly, one of the components of AP-1 turned out to be encoded by jun.

It has recently been determined that the Jun oncoprotein may form a homodimer (Jun-Jun) but preferentially forms a Fos-Jun heterodimer with increased binding affinity for the AP-1 DNA consensus sequence, TGACTCA. Fos and Jun oncoproteins dimerise because both carry "leucine zipper" amino acid structures (leucines at seven residue intervals) in alpha helical regions. The contact surface for DNA binding is adjacent to these zipper domains and dimerisation constrains the FosJun structure for proper attachment to DNA.

The myc oncogene family (c-, L-, and N-myc) also have leucine zipper domains and appear to be nuclear transcription factors. Of particular interest has been the activation of the proto-oncogene myc by chromosomal translocations, usually via a reciprocal $t(8 ; 14)$. This has been found to occur in about $80 \%$ of cases of Burkitt's lymphoma where during B cell differentiation, a translocation juxtaposes myc from chromosome 8 to the immunoglobulin heavy chain (IgH) locus at chromosome 14 resulting in myc activation by a "head to head" IgH/myc gene fusion. A similar chromosomal activation of the c-abl protooncogene has been noted in chronic myelogenous leukaemia where a reciprocal $t(9 ; 22)$ rearrangement places c-abl under the control of a gene called bcr (breakpoint cluster region) resulting in a fusion oncoprotein, p210 bcr/c-abl.

Whereas jun and fos are primary targets for cell signal transduction and act as transcriptional activators via the formation of transcription complexes such as AP-1, Jun-Fos, etc, other nuclear oncogenes have different mechanisms of gene interaction. The erbA oncoprotein up or down regulates a variety of genes by interacting with their promoter 
regions on thyroid hormone binding.

One of the most exciting developments in oncogene research has been the discovery of the so called antioncogenes or recessive oncogenes, the prototype being the retinoblastoma (RB) gene at 13q14 which encodes a nuclear localised DNA binding protein, $\mathrm{p} 105^{\mathrm{RB}}$, that may regulate transcription of certain genes involved in growth control. Individuals with heritable retinoblastoma carry one germ line mutant $\mathrm{RB}^{-}$allele and develop a retinal tumour when the corresponding normal allele is deleted or mutated, thus rendering homozygosity for RB- $^{-}$. Several DNA tumour viruses have been found to produce nuclear oncoproteins that can bind p $105^{\mathrm{RB}}$ leading to neoplastic transformation. The search is now on for other antioncogenes (also called tumour suppressor genes) that by deletion or inactivation may produce malignant transformation. Chromosomal aberrations are prime suspects in tumour induction by antioncogene deletion and in several cases have served to localise the search for such tumour or oncosuppressor genes.

The proto-oncogenes of normal cells are highly regulated in their expression whereas oncogene expression in neoplastic cells is usually constitutive and amplified. It is thought that each type of tumour may express a unique repertoire of activated protooncogenes (oncogenes). Thus it may not be possible to assign inevitable roles in oncogenesis to individual oncogenes. In most cases neoplastic transformation appears to result from a cooperative effect of certain oncogenes (ras and myc, for example) or oncogenes and promoters that may be tissue and carcinogen dependent. The discovery of antioncogenes adds to this complexity of interaction. The growing diversity of regulatory points for the actions of oncogenes suggests that multiple and synergistic oncogenic pathways exist.

In occupational settings chemical genotoxicity presents a likely route of proto-oncogene activation either directly by DNA adduct formation and point mutation or indirectly by chromosomal rearrangements. Moreover, chemical epigenetic toxicity may potentiate oncoprotein derangements. It appears that benzene and toluene and possibly other chemicals may activate protein kinase $\mathrm{C}$ in a manner similar to known tumour promoters such as the phorbol ester TPA. ${ }^{14}$ As noted above, ras oncogenes are often activated in experimental carcinogen induced tumours and the altered $\mathrm{p} 21^{\text {ras }}$ is found in transformed cells. Monoclonal antibodies have been prepared against a variety of oncoproteins including p $21^{\text {ras }}$ and the use of such antibodies as probes for oncoprotein markers has been advocated..$^{15}$

The relation of chemical interactions with DNA and subsequent oncogene activation is not yet clearly defined. DNA is known to undergo spontaneous depurination at about 10000 bases/cell/day and depyrimidination at about 100 bases/cell/day. ${ }^{16} \mathrm{We}$ do not know how carcinogens and aging operate on background rates of genomic instability and intrinsic $\overrightarrow{\overline{\mathrm{C}}}$ somatic mutation. There is considerable variation between individuals in the metabolic activation of chemicals to DNA reactive electrophiles and there $\overline{\bar{c}}$. also appears to be person to person variation in DNA repair processes. ${ }^{17}$ The challenge will be to identify $\stackrel{\circ}{\circ}$ accessible markers of oncogene activation that will $\%$ aid in clarifying the pathogenesis and risk of $\vec{\circ}$ occupational cancer. The pace of discovery in mole- $\overrightarrow{-}$ cular oncology shows no sign of abating and occu- $\vec{\omega}$ pational medicine already faces an embarrassment of $O$ riches in terms of available molecular tools. It is $\frac{\mathbb{D}}{3}$ certainly not too early to begin evaluating this knowledge and technology in terms of the epidemiology, pathogenesis, and prevention of occu- $\dot{i}$ pational cancer. The oncogene era has already $\vec{V}$ provided tantalising glimpses into the mysteries of $\mathrm{O}$ carcinogenesis and promises further revelations that $\rightarrow$ are fraught with implications for our practice of $D$ occupational medicine.

Department of Community Health Sciences

University of Calgary

3330 Hospital Drive NW

Calgary, Alberta

Canada, T2N 4N1

1 Farber E. Possible etiologic mechanisms in chemical carcinogenesis. Environ Health Perspect 1987;75:65-70.

2 Heubner RJ, Todaro GJ. Oncogenes of RNA tumour viruses as $\overrightarrow{+}$ determinants of cancer. Proc Natl Acad Sci USAO 1969;64:1087-94.

3 Weinstein IB. Molecular cancer epidemiology: the use of new laboratory methods in studies on human cancer causation. In: Gordis L, ed. Epidemiology and health risk assessment. Oxford: 응 Oxford University Press, 1988:159-65.

4 Taylor JA. Oncogenes and their applications in epidemiologic $\overparen{\Phi}$ studies. Am J Epidemiol 1989;130:6-13.

5 Rous P. A sarcoma of the fowl transmissible by an agent ${ }^{\circ}$ separable from the tumour cells. $J \operatorname{Exp} M e d 1911 ; 13: 397-411$.

6 Klein G, Klein E. Evolution of tumours and the impact of molecular oncology. Nature 1985;315:190-5.

7 Collett MS, Erikson RL. Protein kinase activity associated with the avian sarcoma virus src gene product. Proc Natl Acad Sci USA 1978;75:2021-4.

8 Hunter T. A thousand and one protein kinases. Cell 1987;50:823-9.

9 Macara IG, Wolfman A. Signal transduction and the ras gene family: molecular switches of unknown function. Trends in Endocrinology and Metabolism 1989;1:26-30.

10 Pai EF, Kabsch W, Krengel U, Holmes KC, John J, Wittinghofer A. Structure of the guanine-nucleotide-binding $N$ domain of the Ha-ras oncogene product $\mathrm{p} 21$ in the triphos- N phate conformation. Nature 1989;341:209-14.

11 Santos E, Nebreda AR. Structural and functional properties of $\sigma$ ras proteins. Federation of American Societies for Experimental Biology Journal 1989;3:2151-63.

12 Landis CA, Masters SB, Spada A, Pace AM, Bourne HR, Vallar L. GTPase inhibiting mutations activate the $\alpha$ chain of $\infty$ $\mathrm{G}_{\mathrm{s}}$ and stimulate adenylyl cyclase in human pituitary tumors. Nature 1989;340:692-6.

13 Vogt PK, Tijan R. Jun: a transcriptional regulator turned oncogenic. Oncogene 1988;3:3-7.

14 DaSilva C, Fan X, Castagna M. Benzene-mediated protein $\varrho$ kinase C activation. Environ Health Perspect 1989;82:91-5.

15 Brandt-Rauf PW, Pincus MR. Oncogenes and oncogene proteins. State Art Rev Occup Med 1987;2:27-38.

16 Loeb LA. Endogenous carcinogenesis: molecular oncology into the twenty-first century. Cancer Res 1989;49:5489-96.

17 Harris CC. Interindividual variation among humans in carcinogen metabolism, DNA adduct formation and DNA repair. Carcinogenesis 1989;10:1563-6. 